become clinical research sites by: alignment with the national priorities; acceleration of current laboratory accreditation processes, improvement of data management, clinical infrastructure, financial management systems; careful recruitment, continuous training and a retention plan of critical research staff; collaborations with private clinical research organisations; marketing of research sites to funders; strengthening institutional review boards; creation of local ownership; and diversification of the research portfolio.

Results Clinical research sites established are Centre Hospitalier Universitaire de Kigali (CHUK) in Kigali city, Centre Hospitalier Universitaire de Butare (CHUB) in Southern province, and Butaro Hospital in Northern province with 26, 26 and 11 dedicated staff, respectively. Sites have minimally a clinical research laboratory under accreditation process, 5 private medical/examination room, a counselling rooms, a data management unit, a waiting area, a pharmacy with restriction-area, administrative area, tele-conference/training room.

Conclusions Development of novel clinical research capacity in resource-limited settings is feasible, with considerable time and resources. Political initiative is a key element for sustainability. Staff retention is the main challenge. For minimising the risk, partnerships between experienced clinical organisations and sponsors are vital for financial stability and knowledge transfer.

\title{
PA-053 ROAD TO BUILDING AND SUSTAINING NOVEL CLINICAL RESEARCH CAPACITY IN RESOURCE-LIMITED SETTINGS: LESSONS LEARNT SO FAR FROM RWANDA
}

Clarisse Musanabaganwa, Jean Pierre Musabyimana, Etienne Karita, Blondin Diop, Abidan Nambajimana, Valentine Dushimiyimana, Prosper Karame, Jim Russell, Jules Ndoli, Prince Bahati, Theobald Hategekimana, Augustin Sendegeya, Jeannine Condo, Agnes Binagwaho. RBC-MRC, Rwanda

\subsection{6/bmjgh-2016-000260.86}

Background Harnessing research for enhancing capacity for evidence-informed policies is key for sustainability in countries that are still facing poverty-related diseases. During current customised medicine and drug-resistance era, priority has shifted to clinical research making clinical trials a powerful tool in availing tailored and affordable drugs and medical interventions. There is need to address disparities in clinical research capabilities worldwide, particularly sub-Saharan Africa, where disease burden is rampant. We share our achievements and lessons learnt so far from establishing three new clinical research sites among the eleven targeted for in Rwanda.

Methods Referral, provincial and specialty hospitals have been selected by the Ministry of Health as potential clinical research sites. Based on the International Conference on Harmonization in Good Clinical Practice (ICH-GCP), a baseline assessment was conducted. Three best-scoring hospitals were developed to 